\title{
HEDGE ALGEBRAS: AN ALGEBRAIC APPROACH TO DOMAINS OF LINGUISTIC VARIABLES AND THEIR APPLICABILITY
}

\author{
Ho N.C. ${ }^{*}$ and Lan V.N. \\ Institute of Information Technology, 18 Hoang Quoc Viet, Hanoi, Vietnam
}

Received 24 October 2005

\begin{abstract}
The paper is an overview on an algebraic approach to domains of linguistic variables and some first applications to show the applicability of this new approach. In this approach, each linguistic domain can be considered as a hedge algebra (HA for short) and based on the structure of HAs, a notion of fuzziness measure of linguistic hedges and terms can be defined. In order to apply hedge algebras to those problems, the results of which are needed, a notion of semantically quantifying mappings (SQMs) will be introduced. It shown that there is a closed connection between SQMs and fuzziness measure of hedge and primary terms (the generators of linguistic domains). To show the applicability of this approach, new methods to solve a Fuzzy Multiple Conditional Reasoning problem, the problem of Balancing an Inverted Pendulum will be presented.
\end{abstract}

\section{INTRODUCTION}

The people do thinking and reasoning to deduce conclusions and to make decision by their own language. Motivated by this, fuzzy sets theory was founded in 1965 by L.A. Zadeh to model human reasoning processes and since then it has been developed intensively and opened several new vast research as well as applied in various areas, in particular in the area of artifitial intelligence. The achievements of fuzzy sets theory in both theoretic and pratical fields are not controversial. However, in order to construct a new approach to human reasoning problem, we have to point out some shortcomings of fuzzy sets theory based approach to this one.

First, in order to establish a computation mechanism for a human reasoning process one has to embed finite linguistic domain of linguistic variables into the set of all functions $F(X,[0,1])$ defined on a universe $X$, that has, as it is well known, a rich computation structure. Based on this, one may study several methods for fuzzy reasoning. So, the structure of human reasoning methods, if it exists and has "may-be no" computation features, is simulated by that of $F(X,[0,1])$, but no one has justified whether such computation methods can model properly the way human do reasoning or not.

On the algebraic point of view, the way we use the whole infinite structure of $F(X,[0,1])$ to model finite domains of linguistic variables is not correct, in our opinion.

Second, it is easy to observe that one can compare meanings of linguistic terms, i.e. one can

* Corresponding author e-mail: ncatho@hn.vnn.vn 
discover an ordering relation on a linguistic domain, based on intuitive meaning of linguistic terms. For example, it is clear that true $\geq$ false, very true $\geq$ more true and approx.false $\geq$ false and so on. However, the mentioned above embedding mapping from this domain into $F(X,[0,1])$ does not preserve the discovered ordering relation!

Third, because we have no way to manipulate directly linguistic terms, it is neccessary in many applications to examine linguistic approximation algorithms, which are usually very complicated.

In our investigation, we shall try to discover algebraic structures of linguistic domains or, in other words, to embed these domains in respective natural algebraic ordered structures in a suitable way so that their elements can be regarded as just linguistic terms. Then, we shall introduce a linguistic reasoning method handling directly linguistic terms. By equipping relatively definite metrics for such algebras, i.e. these metrics must satisfy certain semantic relationships between linguistic hedges, we can examine new methods for multiple conditional fuzzy reasoning, that produce more accurate results than that fuzzy sets-based methods do.

\section{HEDGE ALGEBRAS OF A LINGUISTIC VARIABLE: A SHORT OVERVIEW}

One of reasons to introduce and investigate HAs (see [9, 11, 12]), a mathematical foundation of our method, is that the structure of fuzzy sets does not preserve the ordering structure of linguistic terms determined by their natural meaning such as true $>$ false, very true $>$ true, very false < false, and so on. In this section we shall describe generally what is a HA of a linguistic variable. In fuzzy control ones use verbal descriptions (i.e. linguistic terms) to model a dependence of one physical variable on another one. We denote by $\operatorname{Dom}(X)$ a set of linguistic terms of the linguistic variable $X$, and it is called a domain of $X$. For example, if $X$ is the rotation speed of an electrical motor and Very, More, Possibly, Little are denoted correspondingly by $V, M, P$ and $L$, then $\operatorname{Dom}(X)=\{$ fast, $V$ fast, $M$ fast, $L P$ fast, $L$ fast, $P$ fast, $L$ slow, slow, $P$ slow, $V$ slow, $\ldots\} \cup\{\mathbf{0 , W}, \mathbf{1}\}$ is a domain of $X$. It can be considered as an algebra $A X$ $=(\operatorname{Dom}(X), C, H, \leq)$, where $H=\{V, L, P, M\}$ is the set of hedges, which can be regarded as oneargument operations, $\leq$ is called a semantic ordering relation on $\operatorname{Dom}(X)$, because it is defined by the meaning of linguistic terms, $C=\{$ fast, slow, $\boldsymbol{W}, \mathbf{0}, \mathbf{1}\}$ with $\boldsymbol{W}, \mathbf{0}, \mathbf{1}$ in $\operatorname{Dom}(X)$ interpreted as the neutral, the least and the greatest ones, respectively. The result of applying an $h \in H$ to an $x \in \operatorname{Dom}(X)$ is denoted by $h x$. We denote by $H(x)$ the set of all $u \in \operatorname{Dom}(X)$ generated algebraically from $x$ by using hedges in $H$. That is every $u$ can be expressed in the form $u=h_{n} \ldots h_{1} x$, where $h_{1}$, $\ldots, h_{n} \in H$.

As pointed out in [9], the structure of $A X$ can be built from semantic properties of terms that may also be expressed in term of the semantic ordering relation $\leq$. Intuitively, it is able to order a term-domain based on the following observations (a formal presentation of HAs can be found in $[11,12])$ :

1) Each term has an intuitively semantic tendency which can be recognised by an ordering relation. Two primary terms of each linguistic variable have reverse semantic tendencies: true has a tendency of "going up", called positive tendency, but false has a tendency of "going down", called negative one. They can be characterized by the ordering relationships $V$ true $>$ true and $V$ false < false or simply by true $>$ false! E.g., for the variable AGE, old is positive and young is negative, since old $>$ young.

2) Further, each hedge has an intuitive semantic tendency, which can be expressed also by an ordering relation. It can be seen that the one hedges increase the semantic tendency of the primary terms (called positive hedges), while the other ones (called negative hedges) decrease this meaning. For example, the inequalities $V$ old $>$ old and $V$ young $<$ young mean that $V$ increases the semantic tendency of both terms "old" and "young" and so $V$ is 
positive. But, the hedge $L$ has a reverse effect and hence it is negative. Denote by $H^{-}$the set of all negative hedges and by $H^{+}$the set of all positive ones under consideration. If both hedges $h$ and $k$ do not belong to the same $\mathrm{H}^{+}$or $\mathrm{H}^{-}$, then they have reverse effect and hence they are said to be converse. In the cotrary, they are said to be compatible. In latter case it may happen that one hedge changes the terms more strongly than the other. For example, $L$ and $P$ are compatible and $L>P$, since $L$ false $>P$ false $>$ false. Note that $I<P$ and $I<M$, where $I$, as an artificial hedge, is the identity, i.e. for any term $x, I x=x$. But, it is obvious that $L$ and $V$ are incompatible, i.e. they are converse!

3) Further, we observe that each hedge has an effect of either increasing or decreasing semantic tendency of any others. So, if $k$ increases the semantic tendency of $h$, we say that $k$ is positive w.r.t. $h$. Conversely, if $k$ decreases the semantic tendency of $h$, we say that $k$ is negative w.r.t. $h$. For example, since the semantic tendency of $L$ is expressed by $L$ true < true, it follows from $V L$ true $<L$ true $<P L$ true, that $V$ is positive but $P$ is negative w.r.t. $L$. Similarly, it is observe that $V$ is negative w.r.t. $P$, but positive w.r.t. $M$ and $\mathrm{V}$, and $\mathrm{L}$ is positive w.r.t. $P$, but negative w.r.t. $V$ and $M$. It can be seen also that the positiveness or negativeness of a hedge w.r.t. another one does not depend on the terms they apply to. That is if $V$ is positive w.r.t. $L$ then for any term $x$ we have: (if $x \leq L x$ then $L x \leq V L x$ ) or (if $x \geq L x$ then $L x \geq V L x)$.

4) An important semantic property of hedges is the so called heredity of hedges, which stems from the fact that each hedge modifies only a little, while preserves the essential meaning of each term. This means, for every $h$ term $h x$ inherits the meaning of $x$. This property may also be formulated in term of ordering relation: if the meaning of $h x$ and $k x$ can be expressed by $h x \leq k x$, then $h^{\prime} h x \leq k^{\prime} k x$, (i.e. $h^{\prime}$ and $k^{\prime}$ preserve and hence they can not change the semantic ordering relationship between $h x$ and $k x)$ and so we have $H(h x) \leq$ $H(k x)$. For example, it can be seen intuitively that from L.true $\leq$ P.true it follows that P.L.true $\leq$ L.P.true, or more generally that $H($ L.true $) \leq H($ P.true $)$.

Now, we can intuitively order any domains of physical linguistic variable linearly. For example, the domain of the variable SPEED of a motor considered above can be ordered as follows: $V$ slow $<M$ slow $<$ slow $<P$ slow $<L$ slow $<L$ fast $<L P$ fast $<P$ fast $<$ fast $<M$ fast $<V$ fast and so on.

Formally, as proved in [11,12], that each linguistic domain can be axiomatized, denoted by $A X=$ $(\operatorname{Dom}(X), C, H, \leq)$, and is called a hedge algebra (HA), and is a complete lattice with unit and zero elements 1, 0 under assumption that $H^{-}+I$ and $H^{+}+I$ are lattices of hedges. Particularly, we have

Theorem 2.1 ([11]): Let $A X=(X, C, H, \leq)$ be a HA. Then, the following statements hold:

(i) If $x \in X$ is a fixed point of an $h$ in $H$, i.e. $h x=x$, then it is also a fixed point of the other ones.

(ii) If $x=h_{n} \ldots h_{1} u$, then there exists an index $i$ such that the suffix $h_{i} \ldots h_{1} u$ of $x$ is a canonical representation of $x$ w.r.t. $u$ (that is $x=h_{i} h_{i-1} \ldots h_{1} u$ and $h_{i} h_{i-1} \ldots h_{1} u \neq h_{i-1} \ldots h_{1} u$ ) and $h_{j} x=x$, for all $j>i$.

(iii) If $h \neq k$ and $h x=k x$ then $x$ is a fixed point. $\square$

For convenience in the sequel, we recall here the criteria for comparing any two elements in $\operatorname{Dom}(X)$ :

Theorem 2.2 ([11]). Let $x=h_{n} \ldots h_{1} u$ and $y=k_{m} \ldots k_{1} u$ be two canonical representations of $x$ and $y$ w.r.t. $u$, respectively. Then there exists an index $j \leq \min \{m, n\}+1$ (here as a convention it is understood that if $j=\min \{m, n\}+1$, then either $h_{j}=I$ for $j=n+1 \leq m$ or $k_{j}=I$ for $j=m+1 \leq n$ ) such that $h_{j^{\prime}}=k_{j^{\prime}}$, for all $j^{\prime}<j$ and 
(1) $x=y$ iff $m=n$ and $h_{j} x_{j}=k_{j} x_{j}$;

(2) $x<y$ iff $h_{j} x_{j}<k_{j} x_{j}$;

(3) $x$ and $y$ are incomparable iff $h_{j} x_{j}$ and $k_{j} x_{j}$ are incomparable.

Theorem 2.3. (Th.4 [11]) Let $H^{-}$and $H^{+}$of $A X=(\operatorname{Dom}(X), C, H, \leq)$ be linearly ordered. Then, we have:

(i) For every $u \in \operatorname{Dom}(X), H(u)$ is a linearly ordered set;

(ii) If $C$ is linearly ordered, then so is $\operatorname{Dom}(X)$. Moreover, if $u \leq v$ and $u$ and $v$ are independent, i.e. $u \notin H(v)$ and $v \notin H(u)$, then $H(u) \leq \mathrm{H}(v)$.

\section{DISTANCE AND FUZZINESS MEASURE OF TERMS IN LINEAR HEDGE ALGEBRAS}

It is worth to emphasise that HAs provide an intuitive basis to define fuzziness and then fuzziness measure of terms and hedges suitably. We hope that a more exact mathematical foundation of these notions will be established in the near future. It is well known that one of the important features of linguistic terms is qualitative characteristic. However in many applications we need quantitative characteristic. Therefore, in this section we shall introduce a notion of fuzziness measure and quantitative semantics of terms, which was examined step by step in [6], [10] and [16]. A function $\rho(x, y)$ from $\operatorname{Dom}(X)$ into [0,1] is said to be a metric in an HA, $A X=$ $(\operatorname{Dom}(X), C, H, \leq)$, if it satisfies the following axioms for all $x, y \in X$ :

Axiom 1. $\rho(x, y) \geq 0$ and $\rho(x, x)=0$.

Axiom 2. $\rho(x, y)=\rho(y, x)$.

Axiom 3. $\rho(x, z)=\rho(x, y)+\rho(y, z)$, for any $x, z$ and $y$ such that either $x \geq y \geq z$ or $x \leq y \leq z$.

Axiom 4. For any $h, k \in H^{+}$or $h, k \in H^{-}, \frac{\rho(h x, x)}{\rho(k x, x)}=\frac{\rho(h y, y)}{\rho(k y, y)}$.

Axiom 3 says the required quantitative model of HAs should be linear. Axiom 4 says the relative modification degrees of $h$ and $k$ do not depend on specific terms $x$ or $y$. It is also practically reasonable.

Let us consider a linear HA, $A X=(X, C, H, \leq)$, where $H=H^{-} \cup H^{+}$, and suppose that $H=\left\{h_{-1}, \ldots\right.$, $\left.h_{-q}\right\}$, where $h_{-1}<h_{-2}<\ldots<h_{-q}$, and $H^{+}=\left\{h_{1}, \ldots, h_{p}\right\}$, where $h_{1}<\ldots<h_{p}$, and $h_{0}=I$.

Definition 3.1. Two linear sets $(U, \leq)$ and $(V, \leq)$ are said to be similar if

(1) There exits a one-to-one mapping $f$ from $U$ onto $V$ such that $f$ preserves either the ordering relation $\leq$ or the reverse one $\leq^{*}$ of $U$, where $\leq^{*}$ means that $x \leq^{*} y$ iff $y \leq x$. That is, $f$ satisfies either $(\forall x, y, z \in U)(x<y<z$ iff $f(x)<f(y)<f(z))$, or $(\forall x, y, z \in U)(x<y<z$ $f(x)>f(y)>f(z))$.

(2) For all $x, y, z \in U, \frac{\rho_{U}(x, y)}{\rho_{U}(y, z)}=\frac{\rho_{V}(f(x), f(y))}{\rho_{V}(f(y), f(z))}$.

Lemma 3.2. Let $c \in C$ and denote by $H[u]$ the set $\{h u: h \in H\}$, for any $u$. Then, for any not fixed points $x, y \in X, H[x]$ and $H[y]$ are similar under the mapping $f:=f\left(h_{i} x\right)=h_{i} y$ and, hence, so are $H[x]$ and $H[c]$.

From (v) of Th.2.2 it follows that if $h u<x=h^{\prime} u<h^{\prime}$ ' $u$, then $H[h u]<H[x]<H\left[h{ }^{\prime} u\right]<H\left[h^{\prime \prime}\right.$ ' $]$. So, 
by Lem.3.2, these sets are similar and proportions of distances between their corresponding elements are equal, by Def.3.1. Therefore, Lem.3.2 provides us a basis for constructing metrics in $X$. However, in applications we prefer to use a mapping $f_{s}$ from $X$ into the set of the nonnegative real numbers such that $\rho(x, y)=\left|f_{s}(x)-f_{s}(y)\right|$, called a quantitative semantic mapping (SQMp) of $X$. So, instead of determining the distance $\rho(x, y)$, we construct a SQMp $f_{s}$ from $X$ into $[0,1]$.

First of all, we introduce an intuitive notion of fuzziness measure of terms, which seems not easily to be defined in the framework of fuzzy sets reasonably. Consider the set $H(x)$ consisting of all elements in $X$ generated from $x$ by using hedges. Semantically, it means that $H(x)$ consists of all vague concepts which still contain a definitive essential meaning of the concept $x$ but not of the others. It will be useful to use the sets $H(x), x \in X$, to model the fuzziness degree of $x$, since they have the following properties:

+ If $x$ is a crisp element such as $\mathbf{0 , 1}$ or $\boldsymbol{W}$, then $H(x)=\{x\}$;

+ If $x=h u$, where $h$ is a hedge (and it means that $x=h u$ is more specific than $u$ ), then $H(h u) \subseteq H(u)$, that seems to correspond to the fact that the more specific a term is, the less fuzziness it is.

+ We have also that $H(u)=\cup\{H(h u): h \in H\}$ and $H(h u) \cap H(k u)=\varnothing$ for any hedges $h$ and $k$.

It suggests us to use the "size" of $H(x)$ to express the fuzziness measure of term $x$. In order to define it, let consider a mapping $f$ from $X$ into the unit interval [0,1], which preserves the semantic ordering relation of $X$. Then, "fuzziness measure" can be defined as follows. By fuzziness measure of term $x$, denoted by $f m(x)$, we mean the diameter of the set $f(H(x))=\{f(u)$ : $u \in H(x)\}$. To illustrate this notion, consider an $H A, A X=(X, C, H, \triangle)$, where $H^{+}+I=\{\mathrm{V}, M, I\}$ with $L>M>I, H^{-}=\{L P, I\}$ with $I<P<L, I$ is identity, and $C=\{0$, False, $\boldsymbol{W}$, True, 1$\}$. Then, the fuzziness measure of $x$ can be figured out in Fig. 1.

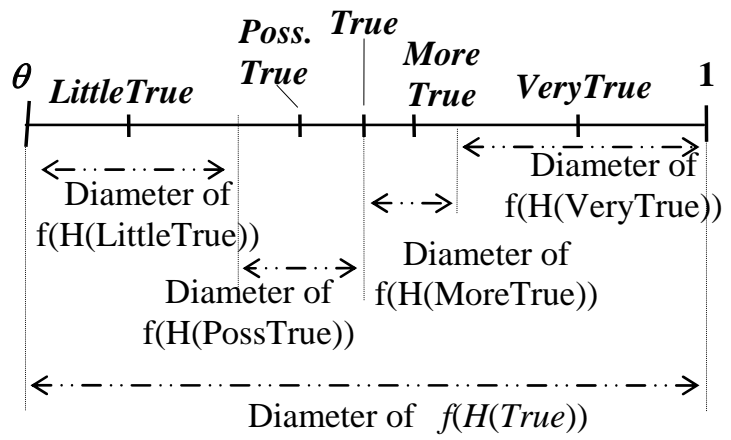

Fig. 1

To establish some constraints of fuzziness measure, we need study the following facts.

Suppose $f m(c)$ is the fuzziness measure of a primary term $c \in\left\{c^{-}, c^{+}\right\}$. Since $c^{-}, c^{+}$have no common meaning, it is natural that $f m\left(c^{-}\right)+f m\left(c^{+}\right) \leq 1$. What do we mean when $f m\left(c^{-}\right)+f m\left(c^{+}\right)<$ 1 ? It means that $\left\{c^{-}, c^{+}\right\}$is not a complete set of primary terms. Since, if $f m\left(c^{-}\right)+f m\left(c^{+}\right)<1$ then it may be understood that there should be still another primary term $c^{\prime}$ different from $c^{-}, c^{+}$so that $f m\left(c^{-}\right)+f m\left(c^{+}\right)+f m\left(c^{\prime}\right) \leq 1$.So, in many applications, we should have $f m\left(c^{-}\right)+f m\left(c^{+}\right)=1$.

Now, we consider a term $u$ and a hedge $h$. The term $x=h u$ is called a particularised term of 
$u$. Consider a term $u=$ good and a set of hedges $\{L, P, M, V\}$. Similarly as above, we find that if there is no more hedges and, hence, the set $\{L$ good, $P$ good, $M$ good, $V$ good $\}$ is "a complete particularisation system" of the term good, then we should have $f m(L$ good $)+f m(P$ good $)+$ $f m(M$ good $)+f m(V$ good $)=1$. In general, if $\{h u: h \in H\}$ is a complete fuzzy particularisation of concept $u$, then we should have $\sum\{f m(h u): h \in H\}=f m(u)$ and we say that $f m$ is a full

measure of the fuzziness of the linguistic terms.

Motivated by this, we give the following definition.

Definition 3.3. $\mathrm{fm}: X \rightarrow[0,1]$ is called a fuzziness measure on $X$, if it satisfies the following conditions:

1) $\mathrm{fm}$ is a full measure on $X$;

2) If $x$ is a crisp concept, i.e. $H(x)=\{x\}$, then $f m(x)=0$. So, $f m(\mathbf{0})=f m(\boldsymbol{W})=f m(\mathbf{1})=0$;

3) For all $x, y \in X$ and $h \in H$, we have $\frac{f m(h x)}{f m(x)}=\frac{f m(h y)}{f m(y)}$, i.e. this ratio does not depend on elements $x$ and $y$ and, hence, it can be denoted by $\mu(h)$ and called the fuzziness measure of hedge h. $\square$

Fuzziness measure on $X$ has the following properties:

Proposition 3.4. For each fuzziness measure $f m$ the following statements hold:

1) $f m(h x)=\mu(h) f m(x)$, for every $x \in X$;

2) $f m\left(c^{-}\right)+f m\left(c^{+}\right)=1$;

3) $\sum_{i=-q, i \neq 0}^{p} f m\left(h_{i} c\right)=f m(c)$, where $c \in\left\{c^{-}, c^{+}\right\}$;

4) $\sum_{i=-q, i \neq 0}^{p} f m\left(h_{i} x\right)=f m(x)$; 5) $\mu$ must satisfy the following equations: $\sum_{i=-1}^{-q} \mu\left(h_{i}\right)=\alpha$ and,$\sum_{i=1}^{p} \mu\left(h_{i}\right)=\beta$ where $\alpha, \beta>0$
and $\alpha+\beta=1$.

Now, it is easy to check the validity of the following:

Theorem 3.5. Let a fuzziness measure $\mu$ of hedges be given such that it satisfies the equalities in 5) of Proposition 3.4 and let $f m\left(c^{-}\right)$and $f m\left(c^{+}\right)$be such that $f m\left(c^{-}\right)>0, f m\left(c^{+}\right)>0$ and $f m\left(c^{-}\right)+$ $f m\left(c^{+}\right)=1$. Then, the mapping $f m$ on $X$ defined recursively by the equation $f m(z)=f m(h x)=$ $\mu(h) f m(x)$, for all $z$ of the form $h x$, and $f m(z)=0$, for $z \in\{\boldsymbol{0}, \boldsymbol{W}, \mathbf{1}\}$, is a fuzziness measure on $X$.

\section{BUILDING SEMANTICALLY QUANTIFYING MAPPINGS OF LINGUISTIC VARIABLES}

On account of the above examination, we have a reasonable way to construct SQMps on linguistic domains.

Definition 4.1. (Sign function). The function Sign: $X \rightarrow\{-1,0,1\}$ is a mapping defined recursively as follows, where the hedges $h$ and $h^{\prime}$ are arbitrary and $c \in\left\{c^{-}, c^{+}\right\}$:

a) $\operatorname{Sign}\left(c^{-}\right)=-1, \operatorname{Sign}\left(c^{+}\right)=+1$,

b) $\operatorname{Sign}\left(h^{\prime} h x\right)=-\operatorname{Sign}(h x)$ if $h^{\prime} h x \neq h x$ and $h^{\prime}$ is negative w.r.t. $h$

(or w.r.t. $c$, if $h=I$ and $x=c$ );

c) $\operatorname{Sign}\left(h^{\prime} h x\right)=\operatorname{Sign}(h x)$ if $h^{\prime} h x \neq h x$ and $h^{\prime}$ is positive w.r.t. $h$

$$
\text { (or w.r.t. } c \text {, if } h=I \text { and } x=c \text { ); }
$$


d) $\operatorname{Sign}\left(h^{\prime} h x\right)=0$ if $h^{\prime} h x=h x$.

Proposition 4.2. For any $h$ and $x$, if $\operatorname{Sign}(h x)=+1$ then $h x>x$, and if $\operatorname{Sign}(h x)=-1$ then $h x$ $<x$.

Definition 4.3 . Let $\mathrm{fm}$ be a fuzziness measure on $X$. A quantitative semantic mapping (SQMp) $v$ on $X$ (associated with $\mathrm{fm}$ ) is defined as follows:

1) $v(\boldsymbol{W})=\theta=f m\left(c^{-}\right), v\left(c^{-}\right)=\theta-\alpha f m\left(c^{-}\right), v\left(c^{+}\right)=\theta \alpha f m\left(c^{+}\right)$;

2) $v\left(h_{j} x\right)=v(x)^{+} \quad \operatorname{Sign}\left(h_{j} x\right)\left\{\sum_{i=1} f m\left(h_{i} x\right)-\omega\left(h_{j} x\right) f m\left(h_{j} x\right)\right\}$, for $1 \leq j \leq p$, and

$$
v\left(h_{j} x\right)=v(x)+\quad \operatorname{Sign}\left(h_{j} x\right)\left\{\sum_{i=-1}^{j} f m\left(h_{i} x\right)-\omega\left(h_{j} x\right) f m\left(h_{j} x\right)\right\} \quad, \quad \text { for }-q \leq j \leq-1,
$$

that can be written in one formula as follows: for $j \in\left[-q^{\wedge} p\right]$, where $\left[-q^{\wedge} p\right]=\{j:-q \leq j \leq p\}$ and $j \neq 0$,

$$
\begin{aligned}
& v\left(h_{j} x\right)=v(x)+\operatorname{Sign}\left(h_{j} x\right)\left\{\sum_{i=\operatorname{sign}(j)}^{j} f m\left(h_{i} x\right)-\omega\left(h_{j} x\right) f m\left(h_{j} x\right)\right\}, \text { where } \omega\left(h_{j} x\right)= \\
& \frac{1}{2}\left[1+\operatorname{Sign}\left(h_{j} x\right) \operatorname{Sign}\left(h_{p} h_{j} x\right)(\beta-\alpha)\right] \in\{\alpha, \beta\}
\end{aligned}
$$

Lemma 4.4. Let $f m$ be a fuzziness measure on $X$. For each SQMp $v$ on $X$ associated with $f m$ defined as above, there exists an $f m$-decomposition system $\mathfrak{I}$ associated with $X$ such that the following statement holds for all $x \in X$ :

$v(x) \in \mathfrak{T}(x)$ and $v(x)$ divides the interval $\mathfrak{T}(x)$ into two subintervals in proportion $\alpha$ to $\beta$. Moreover, if $\operatorname{Sign}\left(h_{p} x\right)=+1$, then the subinterval of the length $\beta f m(x)$ is greater than the other one of the length $\alpha f m(x)$; And if $\operatorname{Sign}\left(h_{p} x\right)=-1$, then the subinterval of length $\beta f m(x)$ is less than the other one.

Proposition 4.5. For all $x \in X, \quad 0 \leq v(x) \leq 1$ and for all $x, y \in X, x<y$ implies $v(x)<v(y)$.

\section{INTERPOLATION REASONING METHOD BASED ON DISTANCE ON HEDGE ALGEBRA}

Let us consider a fuzzy model: $\quad$ if $\quad X=A_{1} \quad$ then $Y=B_{1}$

$$
\text { if } \quad X=A \quad \text { then } Y=B_{n}
$$

where $A_{i}$ and $B_{i}, i=\overline{1, n}$ are verbal descriptions of physical variables $X$ and $Y$, respectively. It is known that each FMCR method provides a mechanism for computing an output $B_{0}$, for an input $A_{0}$.

The effectiveness of each method in applications depends in general on several factors such as fuzzy sets expressing verbal descriptions; implication operators in interpreting the semantics of if-then statements; the aggregation of the obtained if-then fuzzy relations to compute the one representing a certain semantics of the fuzzy model (5.1); the manner of computing composition rule to produce fuzzy output for a given input fuzzy set $A_{0}$; the method of defuzzification. So, although the general idea of FMCR methods is simple, these factors make the methods very difficult to handle and to recognise their behaviour and so make engineers losing intuition in determining effectively methods in applications.

Another method which is more suggestive for solving the above problem is to apply the fuzzy interpolation theory, which investigated firstly by Koczy et al [3,4] and then by Y. Shi, M. 
Misumoto and Z.Q. Wu [13 - 15] and W.H. Hsiao et al [2] and so on. However, these methods seem still to be complicated, e.g. one should apply traditional interpolation methods (INTMd) at each $\alpha$-level of fuzzy sets to compute the output; or some restrictions on fuzzy sets under consideration must be taken to ensure that output results of the method are also, for example, a triangular or normal fuzzy set; or also another restriction is that the fuzzy rules bases must be sparse.

To show the applicability of HAs, we apply traditional INTMd based on quantifying the structure of these algebras to solve FMCR problems. The idea is simply as follows:

Since the fuzzy model (5.1) describes a dependency of $Y$ on $X$, we can regard every if-then statement as a point and hence the given fuzzy model describes a linguistic curve $C$ in $X \times Y$, where $X=\operatorname{Dom}(X)$ and $Y=\operatorname{Dom}(Y)$ are considered as HAs. So, the FMCR problem above can be considered as "a linguistic INTPr" for the curve $C$. Therefore, if we can define distances in $X$ and $Y$, or, equivalently, certain quantitative semantic mappings (SQMps) from $X$ and $Y$ into $[0,1]$, we are able to transform $C$ to a real curve $C_{r}$ in $[0,1] \times[0,1]$ and, then, apply usual INTMds to $C_{r}$ to compute real value output results.

In Section 2 we shall give a short overview on linear HAs. The notion of distances and fuzziness measure of terms will be introduced and investigated in Section 3. In Section 4 we shall give a definition of SQMps and establish a way to compute SQMps for given fuzziness measure of hedges and primary terms. A method of interpolation to solve FMCR problems is presented in Section 5. In order to show besides the effectiveness of the new method, we shall re-examine the same examples examined in [1].

Our method has some advantages. (i) Based on Hedge Algebras of term-domains, it is able to define suitably fuzziness degree of terms and, especially, hedges and quantitative semantic mappings (SQMp) of a term-domain; (ii) FMCR Pr. can be solved by classical INTMds and it seems to be much more suggestive, simple and produces more accurate and real-value results, i.e. defuzzification is not necessary; (iii) Because SQMps are one-to-one, linguistic approximation problems become simple. Note that fuzziness measure of hedges and primary terms, and especially the real value $\theta$ of the neutral element, which is chosen arbitrarily in $[0,1]$, considered as the parameters will make the method also flexible.

In fuzzy control, we often deal with FMCR problems. The physical variables of these problems are normally modelled by linguistic variables, whose real domains usually are linearly ordered sets. So, HAs as models of physical variable must be linear sets as well. This suggests us to deal with a new INTMd to solve FMCR problems, based on quantitative semantic mappings (SQMps) examined above.

Consider a fuzzy model (5.1). Using fuzzy sets-based methods in fuzzy multiple conditional reasoning, we should carry out many tasks: To determine an appropriate reasoning method (a generalised Modus Ponens) or a fuzzy interpolation reasoning methods ([2 - 4, 13 - 15])); To determine fuzzy sets, membership functions of which should represent suitably the meaning of terms; To find a reasonable defuzzification method; and so on. Since the results depend on several factors, using these methods ones lose intuition and encounter difficulties to recognise their behaviour.

Here we introduce a more suggestive approach based on INTMds. The idea is as follows: For a given fuzzy model as (5.1), we interpret each if-then statement of this model as defining a point and, therefore, this model defines a fuzzy curve $C_{f}$ in the Cartesian product $X \times Y$, where $X$ and $Y$ are considered as HAs of the linguistic variables $X$ and $Y$, respectively. Then, the FMCR problem saying that "For a given fuzzy model (5.1) and an input $A$, find an output $B$ corresponding to $A$ " may be understood as an INT Problem for the given fuzzy curve $C_{f}$ in $X \times Y$.

So, the main steps of our method simply are the following: 
1) Construct SQMps $v_{X}$ and $v_{Y}$, which map $X$ and $Y$ into the interval [0,1], respectively. These mapp-ings are computed by Def.4.3 and, by Th.3.5, based on users parameters $\mu(h)$ with $h \in H$, $\mu\left(c^{+}\right)$and $\theta$.

2) Under mappings $v_{X}$ and $v_{Y}$, the fuzzy curve $C_{f}$ in $X \times Y$ is transformed into a real curve $C_{r}$ in $[0, a] \times[0, b]$, where $[0, a]$ and $[0, b]$ are the given domains of the basic variables of $X$ and $Y$, respectively.

3) Aply linear INTMd to the obtained $C_{r}$ curve to compute the output corresponding to a given input.

To evaluate the method, we have studied the same 7 examples examined in [1], fuzzy models of which are given in the Appendix. The maximal model error of these fuzzy models has been defined in [1] to be equal to 400, while our maximal model error defined in [5] is equal 200.

For each fuzzy model, using our method the corresponding output values of $N$, for the given real values of $I$ are computed and the results are given in row No. 6 of Tab.1. The maximal errors are two large in EX2 (Error = 800) and rather large (greater than 300) in EX1, 3, despite our effort to find several systems of parameters $\theta, \mu\left(c^{+}\right)$and $\mu(h)$ of hedges. The reason of this may due to the fact that the fuzzy models describing real world curves are not appropriate, because according to our intuition the right-end triangular $\mu_{\text {VLarge }}$, with $\mu_{\text {VLarge }}(2000)=1$, defined on the basic variable domain [400,2000] of $N$ for ' $V$ Large', should represent a meaning of another term which is clearly greater than VLarge, e.g. it is ' $V V$ Large'. A similar comment can be made for the terms Null and Zero. Therefore, before performing our method, unsuitable linguistic descriptions in the fuzzy models should be changed as follows:

Null:=VV Small, Zero: $=V$ M Small, $V$ Large:= $V$ V Large. The other verbal descriptions remain unchanged.

\section{Table 1}

\begin{tabular}{|c|c|c|c|c|c|c|c|c|}
\hline & Methods & EX1 & EX2 & EX3 & EX4 & EX5 & EX6 & EX7 \\
\hline 1 & Max error of Cao-Kandel method with operator $5^{*}$ & 200 & 300 & 400 & 400 & 400 & 80 & 400 \\
\hline 2 & Max error of Cao-Kandel method with operator $22 *$ & 200 & 350 & 400 & 400 & 400 & 80 & 200 \\
\hline 3 & Maxi error of Cao-Kandel method with operator 8 & 300 & 350 & 200 & 150 & 200 & 100 & 200 \\
\hline 4 & Max error of Cao-Kandel method with operator 25 & 300 & 400 & 200 & 200 & 200 & 100 & 200 \\
\hline 5 & Max error of Cao-Kandel method with operator 31 & 300 & 400 & 200 & 200 & 200 & 100 & 200 \\
\hline 6 & $\begin{array}{l}\text { Maximal error caused by our method with } \\
\theta=0.5, \alpha=0.4, \mu(L)=\mu(P)=0.2, \mu(M)=\mu(V)= \\
0.3, \text { for } I\end{array}$ & 353 & 800 & 412 & 229 & 248 & 200 & 229 \\
\hline 7 & $\begin{array}{l}\text { Maximal error caused by our method with } \\
\theta=0.628, \alpha=0.4, \mu(L)=\mu(P)=0.2, \mu(M)=\mu(V) \\
=0.3, \text { for } I\end{array}$ & 254 & 228 & 104 & 104 & 77 & 66 & 104 \\
\hline 8 & $\begin{array}{l}\text { Maximal error caused by our method with } \\
\theta=0.628, \alpha=0.4, \mu(L)=0.22, \mu(P)=0.18, \mu(M)=0.28 \\
\mu(V)=0.32 \text {, for } I\end{array}$ & 236 & 197 & 102 & 80 & 77 & 47 & 80 \\
\hline
\end{tabular}

The second negative effect is causing by parameter $\theta$. Usually, ones choose $\theta=1 / 2$. However we 
recognise that it depends on the shape of the curve under consideration. For example, if we change the value of $\theta$ to be 0.628 and $\mu(h)$ of hedges of variable $I$ are chosen the same as those given in row No.6 and the user parameters for $N$ are chosen the same in all examples. The results given in row No 7 of Tab.1 are obviously much improved. Note that the implication operators $5 *, 22 *, 8,25$ and 31 were shown in [1] to be the best applicability ones.

Comparing the computing results in rows No 7 and 8, it is shows that fuzziness measure of hedges also have an significant influence on the output results.

\section{ALGORITHM OF CONTROL BASED ON HEDGE ALGEBRAS}

Let us consider a general Fuzzy Associate Memory (FAM) in the following form:

If $X_{1}=A_{11}$ and $\ldots$ and $X_{m}=A_{1 m}$ then $Y=B_{1}$

If $X_{1}=A_{21}$ and $\ldots$ and $X_{m}=A_{2 m}$ then $Y=B_{2}$

If $X_{1}=A_{n 1}$ and $\ldots$ and $X_{m}=A_{n m}$ then $Y=B_{n}$

where $A_{i j}$ and $B_{i}, i=1, \bar{n}$ and $j=1, m$ are verbal descriptions of physical variables $X_{j}$ and $Y$, respectively. In fuzzy control, these verbals, which are linguistic terms, are regarded as labels of designed fuzzy sets, which describe real values of variables.

However, computations based on fuzzy sets seem to be complecated and loosing intuition. Based on hedge algebras approach, these labels are regarded as just linguistic terms and, by SQMs, viewed as just real values of variables. Therefore, hedge algebras may provide simpler computation.

Based on this new idea, we introduce a general fuzzy control model based on the theory of hedge algebras, called Hedge Algebras-based Controller (HAC). Figure 2 shows the general schema of HAC, where $r$ is reference, $e$ is error and $u$ is control action, $P$ is plant.

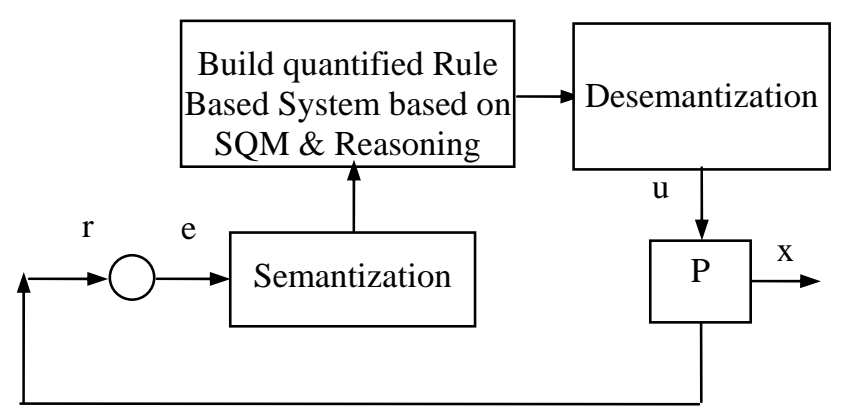

Fig. 2: Hedge Algebras-based Controller

Computing process of the control algorithm is illustrated in Fig.3 

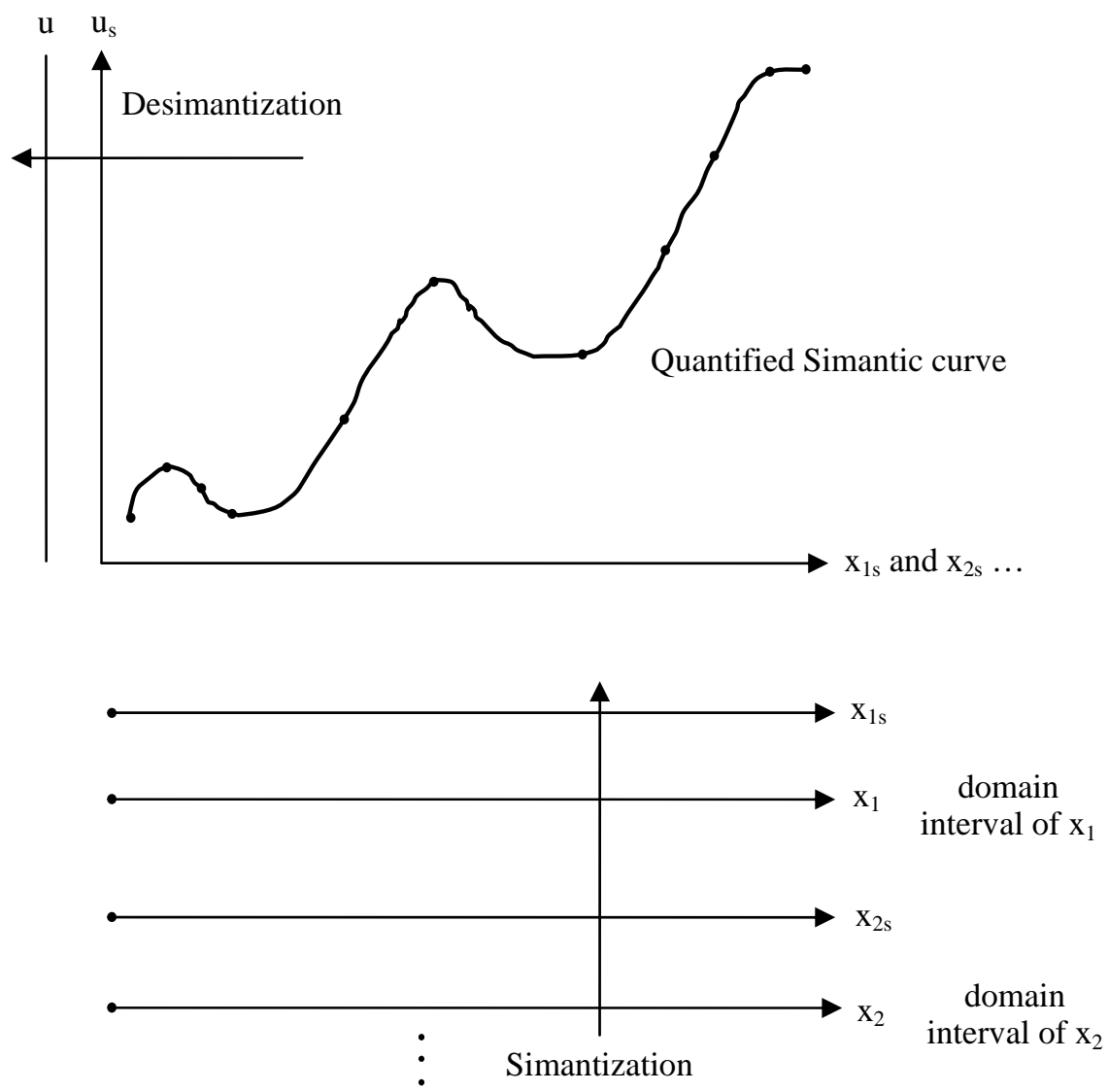

Fig. 3: Computing process of the HAC algorithm

Because the basis knowledge data are given by FAM, we should define SQMs, which map terms into a semantic range of $[0,1]$. To comput this value, we choose a suitable semantic operating range [s0,s1] and determine carefuly a universe of discourse (domain interval ) of the considered variable.

Step 2 : Build quantified Rule Based System based on SQM \& Reasoning.

Using SQMs defined in Step 1, transform FAM into a table with numerical data, called Semantical Associate Memory (SAM) and define the quantified semamtic curve by an interpolation function.

Step 3: Desemantization.

It simply a mapping which assigns each semantic value in the semantic range of the control action with a real value in the operating range.

From Step 1 to Step 3 one can see that the proposed algorithm for fuzzy control is more simple than conventional one in the sence that it does not require defuzzification strategy, since it requires a careful choice of:

1. The number of membership functions

2. The shape of membership functions

3. The definition of fuzzy implication 
4. A measure of central tendency (center of mass) of the membership functions;

Hence, Hedge Algebras approach has some advantages over the fuzzy approach, namely, it quick to be constructed, more intuitive and more exact.

To explain these, we reconsider one of classical problems which has been an interesting case in the study of nonlinear systems for many years, it is the inverted pendulum. The control problem is to regulate the position of the pendulum. The differential equation describing the simplest inverted pendulum in [28] is given below:

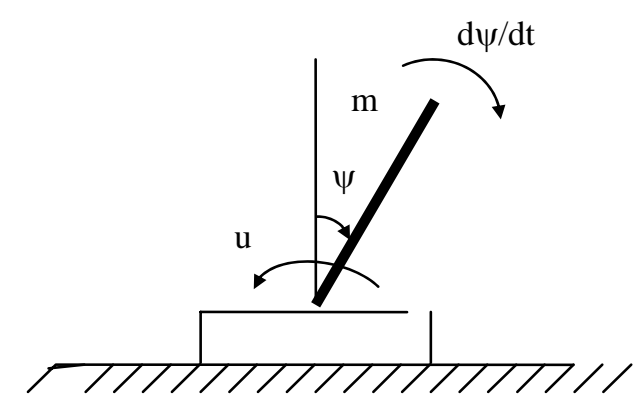

Fig. 4: Inverted pendulum control problem

$$
-m l^{2} \mathrm{~d}^{2} \psi / \mathrm{dt}^{2}+\operatorname{mlg} \sin \psi=\mathrm{u}(\mathrm{t})
$$

where :

$m$ is the mass of the pole located at the tip point of the pendulum

$l \quad$ is the lenght of the pendulum

$\Psi$ is the deviation angle from vertical in the clockwise direction

$u(t)$ is the torque aplied to the pole in the counterclockwise direction $-(u(t)$ is the control action)

$t$ is time

$g$ is the gravitational acceleration constant

Assuming that $x_{1}=\Psi$ and $x_{2}=d \Psi / d t$ are state variables. The state-space representation for the nonlinear system defined by (6.2) is given by

$$
\begin{aligned}
& d x_{1} / d t=x_{2} \\
& d x_{2} / d t=(g / l) \sin x_{1}-\left(1 / m l^{2}\right) u(t)
\end{aligned}
$$

It is known that for very small rotation, or $\Psi$, we have $\sin \Psi=\Psi$, where $\Psi$ is measured in radians. This relation is used to linearize the nonlinear state-space equation and we get

$$
\begin{aligned}
& d x_{1} / d t=x_{2} \\
& d x_{2} / d t=(g / l) x_{1}-\left(1 / m l^{2}\right) u(t)
\end{aligned}
$$

If $x_{1}$ is measured in degrees and $x_{2}$ is measured in degrees per second, by choosing $l=g$ and $m=180 / \pi g^{2}$, the linearized and discrete state-space equations can be represented as matrix difference equation

$$
x_{1}(k+1)=x_{1}(k)+x_{2}(k)
$$




$$
x_{2}(k+1)=x_{1}(k)+x_{2}(k)-u(k)
$$

For this problem we assume that the universe of discourse for the two variables are as follows:

$$
-2^{0} \leq x_{1}(k) \leq 2^{0} \quad ; \quad-5 d p s \leq x_{2}(k) \leq 5 d p s \quad \text { (dps=degree per second) }
$$

and the universe of discourse for the control is $-24 m A \leq u(k) \leq 24 m A$.

The goal of the controller design is to seek a control signal $u$ that will keep the inverted pendulum just in or closely to the vertical stable position ( i.e. it is defied by $e=0$ and $\Delta e=0$ ). To synthesize a controller using hedge algebras we, firstly determine a common SQM for both variables $e$ and $\Delta e$ as follow:

Let $A X=(X, C, H+\cup H-, \triangleleft)$ be a hedge algebra, where

$C=\{0$, Small, $\theta$, Large, 1$\}$

$H=\{$ Little $\}=\left\{h_{-1}\right\} ; q=1$

$H^{+}=\{$Very $\}=\left\{h_{1}\right\} ; p=1$

Assume that $\theta=0.5$ and the fuzziness measure of primary terms and hedges are give by

$$
\begin{aligned}
& \mu(\text { Very })=0.5=\mu\left(h_{1}\right) ;(\beta=0.5) \\
& \mu(\text { Little })=0.5=\mu\left(h_{-1}\right) ;(\alpha=0.5)
\end{aligned}
$$

$f m($ Small $)=\theta=0.5$

$f m($ Large $)=1-f m($ Small $)=1-0.5=0.5$

So, SQM is defined by the following recursive formulaes:

1) $\quad v($ Small $)=\theta-\alpha f m($ Small $)=0.5-0.5 \times 0.5=0.25$

2) $(j=1) ; v($ Very Small $)=v($ Small $)+$ Sign $($ Very Small $) x$

$$
\left\{\sum_{\mathrm{i}=1}^{1} f m\left(h_{\mathrm{i}} \text { Small }\right)-0.5 f m\left(h_{1} \text { Small }\right)\right\}=0.25+(-1)\{0.5 \times 0.5-0.5 \times 0.5 \times 0.5\}=0.125
$$

3) $(j=-1)$; $v($ Little Small $)=v($ Small $)+$ Sign $($ Little Small $) x$

$\left\{\sum_{i=-1}^{-1} f m\left(h_{i}\right.\right.$ Small $)-0.5 f m\left(h_{-1}\right.$ Small $\left.)\right\}=0.25+(+1)\{0.5 \times 0.5-0.5 \times 0.5 \times 0.5\}=0.375$

4) $\quad v($ Large $)=\theta+\alpha f m($ Large $)=0.5+0.5 \times 0.5=0.75$

5) $\quad(j=1) ; v($ Very Large $)=v($ Large $)+\operatorname{Sign}($ Very Large $) x$

$\left\{\sum_{\mathrm{i}=1}^{1} \mathrm{fm}\left(\mathrm{h}_{\mathrm{i}} L \arg e\right)-0.5 \mathrm{fm}\left(h_{1} L \arg e\right)\right\}=0.75+(+1)\{0.5 \times 0.5-0.5 \times 0.5 \times 0.5\}=0.875$

6) $(j=-1) ; v($ Little Large $)=v($ Large $)+$ Sign $($ Little Large $) x$

$\left\{\sum_{i=-1}^{-1} f m\left(h_{i}\right.\right.$ Large $)-0.5 f m\left(h_{-1}\right.$ L arge $\left.)\right\}=0.75+(-1)\{0.5 \times 0.5-0.5 \times 0.5 \times 0.5\}=0.625$

To start the simulation by the algorithm using hedge algebras, we will use the following crisp initial conditions given by $e^{o}=x_{1}(0)=1^{0}$ and $\Delta \mathrm{e}^{0}=x_{2}(0)=-4 d p s$

Step 1: Construction quantified semantic intervals based on domain intervals of $e, \Delta e$ and $u$. 


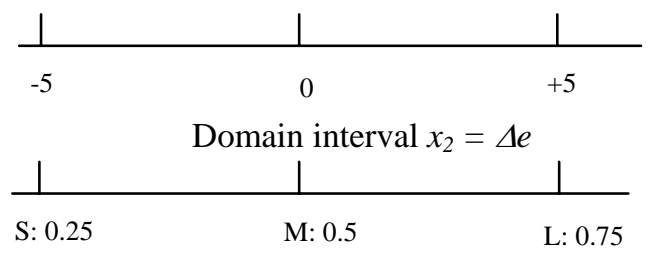

Quantified semantic interval $x_{2 s}=\Delta_{e s}$
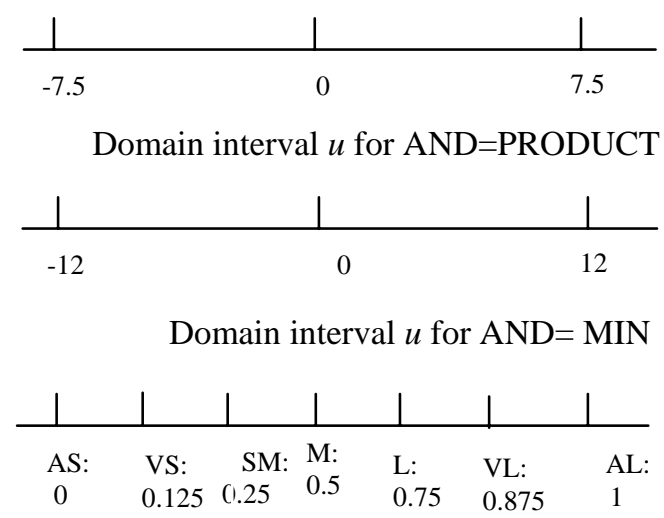

Quantified semantic interval $u_{s}$

Step 2: Determine the semantic value of hedge of each corresponding IF-THEN statement from the FAM table of [28].

Table 2: Semantic Associative Memory

\begin{tabular}{|c|c|c|c|}
\hline $\mathrm{L}: 0.75$ & $\mathrm{VL}: 0.875$ & $\mathrm{~L}: 0.75$ & $\mathrm{M}: 0.5$ \\
\hline $\mathrm{M}: 0.5$ & $\mathrm{~L}: 0.75$ & $\mathrm{M}: 0.5$ & $\mathrm{~S}: 0.25$ \\
\hline $\mathrm{S}: 0.25$ & $\mathrm{M}: 0.5$ & $\mathrm{~S}: 0.25$ & $\mathrm{VS}: 0.125$ \\
\hline
\end{tabular}

Define the quantified semantic curve by an interpolation function over semantic points.

We have calculated the Cartesian product of two input quantified semantic values using two cases, in which the first is AND=PRODUCT and the second is AND=MIN to get the input real values in $[0,1]$.

The quantified semantic curve can be represented by the line over semantic points as shown in Figs. 5 and 6. 


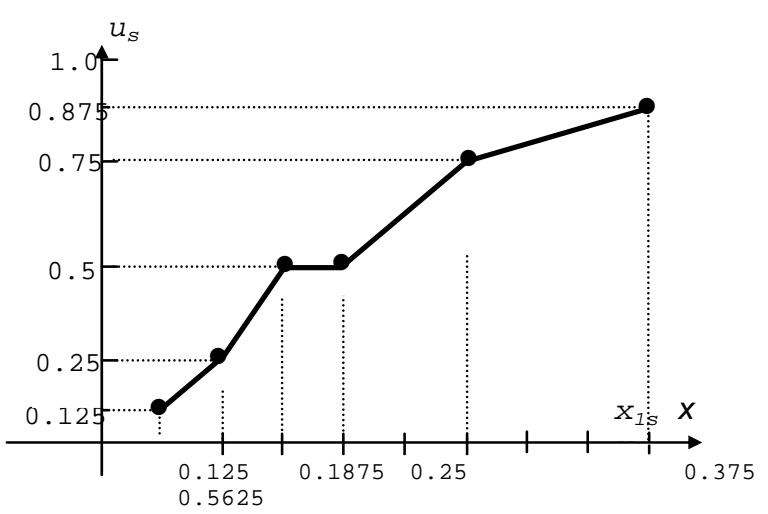

Fig. 5 : Quantified semantic curve for $A N D=P R O D U C T$

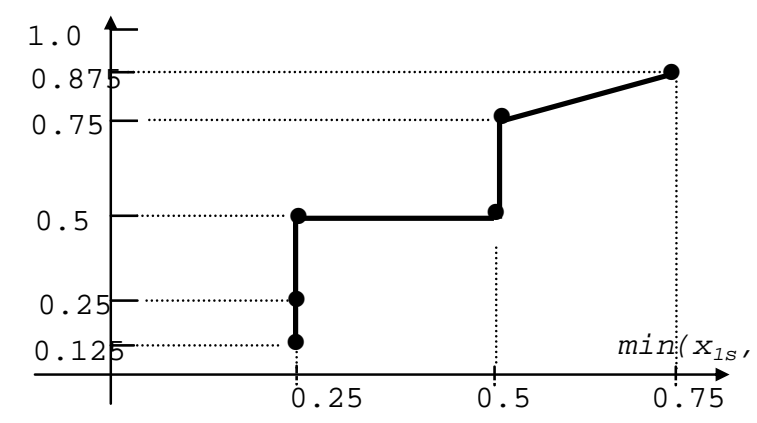

Fig. 6 : Quantified semantic curve for $A N D=M I N$

Table 3: The simulation results of HAC and FC

\begin{tabular}{|c|c|c|c|c|c|c|c|c|c|}
\hline \multicolumn{7}{|c|}{ Hedge Algebras-based Controller } & \multirow{2}{*}{\multicolumn{3}{|c|}{$\begin{array}{l}\text { Fuzzy Conventional } \\
\text { Controller[28] }\end{array}$}} \\
\hline \multicolumn{4}{|c|}{$A N D=P R O D U C T$} & \multicolumn{3}{|c|}{$A N D=M I N$} & & & \\
\hline$k$ & $x_{1}(k)$ & $x_{2}(k)$ & $u(k)$ & $x_{1}(k)$ & $x_{2}(k)$ & $u(k)$ & $x_{1}(k)$ & $x_{2}(k)$ & $u(k)$ \\
\hline 0 & 1 & -4 & -1 & 1 & -4 & 0 & 1 & -4 & -2 \\
\hline 1 & -3 & -2 & -6 & -3 & -3 & -12 & -3 & -1 & -9.6 \\
\hline 2 & -5 & 1 & -4 & -6 & 6 & 0 & -4 & 5.6 & 0.0 \\
\hline 3 & -4 & 0 & -5 & 0 & 0 & 0 & 1.6 & 1.6 & 5.28 \\
\hline 4 & -4 & 1 & -4 & 0 & 0 & 0 & 3.2 & -2.08 & 1.12 \\
\hline 5 & -3 & 1 & -4 & 0 & 0 & 0 & 1.12 & 0 & 4.32 \\
\hline 6 & -2 & 2 & 0 & 0 & 0 & 0 & 1.12 & -3.2 & 0.8 \\
\hline 7 & 0 & 0 & 0 & 0 & 0 & 0 & -2.08 & -2.28 & -9.86 \\
\hline 8 & 0 & 0 & 0 & 0 & 0 & 0 & -4.36 & 5.5 & 0.0 \\
\hline 9 & 0 & 0 & 0 & 0 & 0 & 0 & 1.14 & 1.14 & 6.8 \\
\hline
\end{tabular}


Step 3: With crisp initial condition $x_{1}(0)=1^{0}$ and $x_{2}(0)=-4 d p s$, the quantified semantic curve will produce the real control action $u(k)$ with $k=0,1,2, \ldots$. Each control action $u(k)$ after $k$ $=0$ will begin with the previous values of $x_{1}$ and $x_{2}$ as the input conditions to the next cycle of the recursive state equations.

All the simulation results for the case AND=PRODUCT and AND=MIN are shown in Table 3. Conventional fuzzy control of [28] is shown also in Table 3.

For the comparison purpose of HAC and FC, we define the error function of control as follows $(\mathrm{r}=0$ and $\Delta \mathrm{r}=0$ )

$$
e(k)=\left[\left(x_{1}(k)-r\right)^{2}+\left(x_{2}(k)-\Delta r\right)^{2}\right]^{1 / 2}
$$

The contrrol error function of the inverted pendulum by HAC and FC is represented in Fig.7.

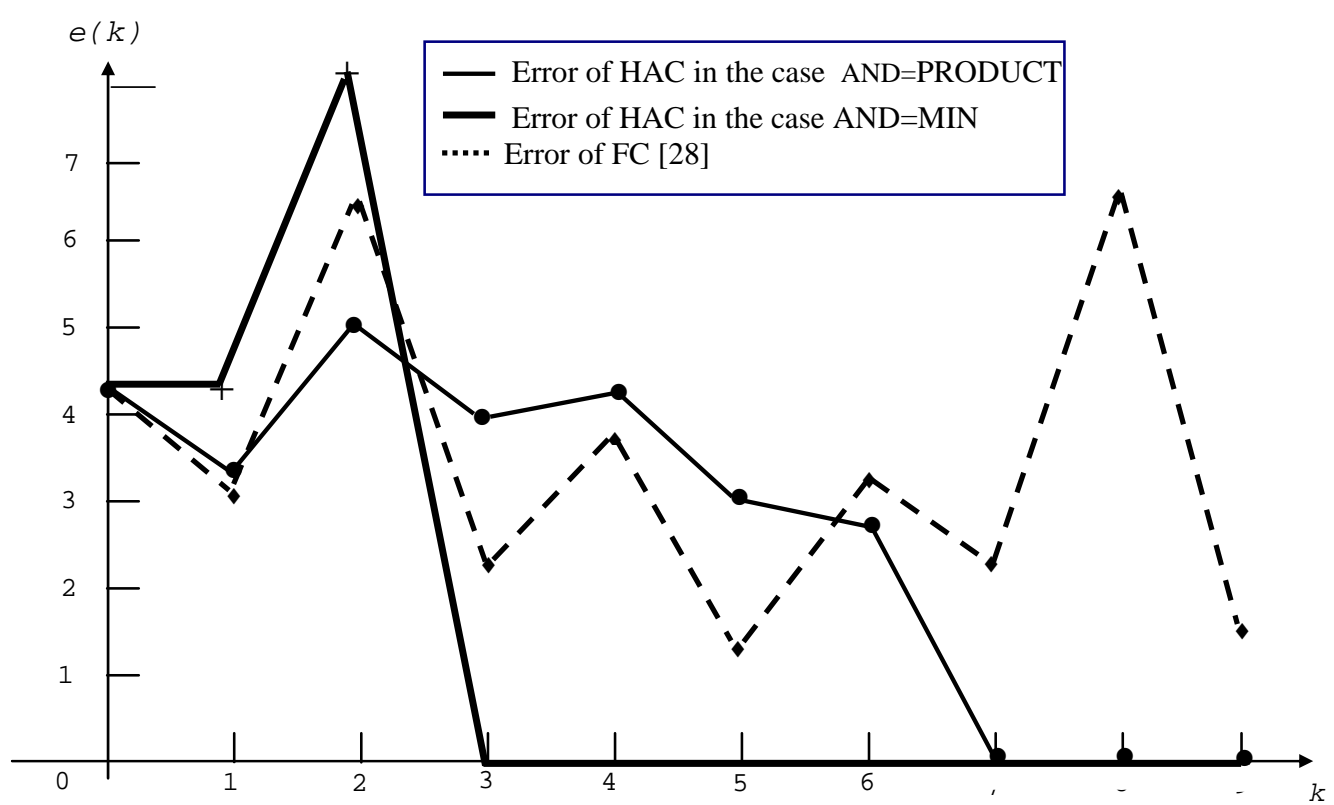

Fig. 7: Error of HAC and FC

\section{CONCLUTIONS}

We have presented an algebraic approach to domain of linguistic variables and some first application. The results of simulation specially in control problems show that the method of control based on Hedge Algebras is simple and exact. We believe that the basic idea behind the approach will have a significant influence on practice of a fuzzy reasoning and control problem in future.

\section{REFERENCES}

1. Cao Z. and Kandel A. (1989), Applicability of some fuzzy implication operators, Fuzzy sets and systems, vol. 31, pp. 151-186.

2. Hsiao, W.H., Chen, S.M., and Lee C.H. (1998), A new interpolative reasoning method in sparse rule-based systems, vol. 93(1).

3. Koczy, L.T. and Hirota, K. (1993), Interpolative reasoning with insufficient evidence in 
sparse fuzzy rules bases, Inform. Sci., vol. 71, pp. 169-201.

4. Koczy, L.T. and Hirota, K. (1993), Approximate reasoning by linear rule interpolation and general approximation, Internat. J. Approx. Reason, vol. 9, pp. 197-225.

5. Nguyen Cat Ho (1997), A consideration of some factors influencing on the accuracy of fuzzy conditional reasoning, Proceeding of Artificial Intelligence and Information-Control System of Robots conference of Slovak (AIICSR 97 Conf,), Bratislava, Sep 10-14.

6. Nguyen Cat Ho and Tran Thai Son (1995), On the distance between values of linguistic variables in hedge algebra, Journal of Computer Science and Cybernetics, vol. 11(1), pp. 10-20 (in Vietnamese).

7. Nguyen Cat Ho and Huynh Van Nam (1999), Ordered Structure-Based Semantics of Linguistic Terms of Linguistic Variables and Approximate Reasoning, AIP Conf. Proceed. on Computing Anticipatory Systems, CASYS’99, $3^{\text {th }}$ Inter. Conf., pp. 98-116.

8. Nguyen Cat Ho and Tran Thai Son (1997), On fuzzy model error, Journal of Informatics and Cybernetics, vol. 13(1) pp. 66-72 (in Vietnamese).

9. Nguyen Cat Ho (1987), Fuzziness in structure of linguistic truth values: a foundation for development of fuzzy reasoning. Proc. of Int. Symp. on Multiple-Valued Logic, May 26-28, 1987, Boston University, Boston, Massachusetts, IEEE Computer Society Press, pp. 325335.

10. Nguyen Cat Ho et al. (1999), HAs, Linguistic-valued Logic and Their Application to Fuzzy Reasoning, Internat. J. of Uncertainty, Fuzziness and Knowledge-Based Systems, vol.7(4), pp. 347-361.

11. Nguyen Cat Ho and Wechler, W. (1990), Hedge algebra: An algebraic approach to structures of sets of linguistic truth values, Fuzzy sets and systems, vol. 35, pp. 281-293.

12. Nguyen Cat Ho and Wechler, W. (1992), Extended algebra and their application to fuzzy logic, Fuzzy sets and systems, vol. 52, pp. 259-281.

13. Shi, Y. and Mizumoto, M. (1997), Reasoning conditions on Koczy's interpolative reasoning method in sparse fuzzy rule bases, Part II, Fuzzy sets and systems, vol. 87, pp. 47-56.

14. Shi, Y. and Mizumoto, M. (1998), A note on reasoning conditions of Kocy's interpolative reasoning method (Short Communication), Fuzzy Sets and Systems, vol. 96, pp. 373-379.

15. Shi, Y., Mizumoto, M. and Wu, Z.Q. (1995), Reasoning conditions on Koczy's interpolative reasoning methods in sparse fuzzy rules bases, Fuzzy Sets and Systems, vol. 75, pp. 63-71.

16. Bezdek J. (1993), Fuzzy models-what are they, and Why ?, IEEE Trans. Fuzzy Systems, vol. 1, pp. 1-5.

17. Cao Z. and Kandel A.(1989), Applicability of some fuzzy implication operators, Fuzzy sets and systems, vol. 31, pp. 151-186.

18. Dubois D. and Prade H. (1996), New trends and open problems in fuzzy logic and approximate reasoning , Theoria, vol. 11(27), pp. 109-121.

19. Hajek P. (1998), Mathematics of Fuzzy logic. Kluwer, Dordrecht .

20. Hsiao, W.H., Chen, S.M., and Lee, C.H. (1998), A new interpolative reasoning method in sparse rule-based systems, vol. 93(1), January 1,

21. Kiszka, J.B., Kochanska, M., and Sliwinska, D.S. (1985), The inference of some fuzzy implication operators on the accuracy of a fuzzy model, part 1, Fuzzy sets and systems, vol. 


\section{5, pp. 111-128.}

22. Kiszka, J.B., Kochanska, M., and Sliwinska, D.S. (1985), The inference of some fuzzy implication operators on the accuracy of a fuzzy model, part 1, Fuzzy sets and systems, vol. 15, pp. 223-240.

23. Klawonn, F. and Novak, V. (1996), The relations between inference and interpolation in frame work of fuzzy systems, Fuzzy Sets and Systems, vol. 81, pp. 336-354.

24. Mamdani, E.H.(1977), Application of fuzzy logic to approximate reasoning using linguistic synthesis, IEEE Transaction on computer, vol. 26, pp. 1182-1191.

25. Mizumoto, M. (1985), Extended fuzzy reasoning, Approximate reasoning in expert systems (M.M. Gupta, A.Kandel, W. Bandler, J.B. Kiszka Ed.), Elsevier Science Publishers NorthHolland.

26. Mizumoto, M. and Zimmermann, H.J. (1982), Comparison of fuzzy reasoning methods, Fuzzy sets and systems, vol. 18, pp. 253-283.

27. Novak, V. (1994), Fuzzy control from logical point of view. Fuzzy Sets and Systems, vol. 66, pp. 154-173.

28. Ross, T.J. (1997), Fuzzy logic with Engineering application. International Edition. Mc Graw-Hill, Inc.

29. Yager, R.R. (1994), Aggregation operators and fuzzy systems modelling. Fuzzy Sets and Systems, vol. 67, pp. 129-145.

30. Zadeh, L.A. (1972), Fuzzy set theoretic interpretation of linguistic hedges. J. Cybernetics, vol. 2, pp. 4-34.

31. Zadeh, L.A. (1975), The concept of linguistic variable and its application to approximate reasoning. Inform. Sci., vol. 8, pp. 199-249.

APPENDIX: Fuzzy models and real data for EX1-EX7 examined in [1]

\begin{tabular}{|c|c|c|c|c|c|c|c|c|c|c|}
\hline \multicolumn{3}{|c|}{ EX1 } & \multicolumn{3}{|c|}{ EX2 } & \multicolumn{3}{|c|}{ EX3 } & \multicolumn{2}{|c|}{ EX4 } \\
\hline Values of I & \multicolumn{2}{|c|}{ Values of $\mathbf{N}$} & \multicolumn{2}{|c|}{ Values of $\mathbf{I}$} & Values of $\mathbf{N}$ & Values of I & \multicolumn{2}{|c|}{ Values of $\boldsymbol{N}$} & Values of $\mathbf{I}$ & Values of $\mathrm{N}$ \\
\hline Null & \multicolumn{2}{|c|}{ Very_Large } & \multicolumn{2}{|c|}{ Null } & Very_Large & Null & \multicolumn{2}{|c|}{ Very_Large } & Null & Very_Large \\
\hline Zero & \multicolumn{2}{|c|}{ Large } & \multicolumn{2}{|l|}{ Zero } & Zero & Zero & \multicolumn{2}{|c|}{ Medium } & Zero & Medium \\
\hline Small & \multicolumn{2}{|c|}{ Medium } & \multicolumn{2}{|l|}{ Small } & Very_Large & Small & \multicolumn{2}{|c|}{ Zero } & Small & Small \\
\hline Medium & \multicolumn{2}{|c|}{ Small } & \multicolumn{2}{|c|}{ Medium } & Zero & Medium & \multicolumn{2}{|c|}{ Medium } & Medium & Small \\
\hline Large & \multicolumn{2}{|c|}{ Zero } & \multicolumn{2}{|c|}{ Large } & Very_Large & Large & \multicolumn{2}{|c|}{ Very_Large } & Large & Zero \\
\hline Very_Large & \multicolumn{2}{|c|}{ Zero } & \multicolumn{2}{|c|}{ Very_Large } & Zero & Very_Large & \multicolumn{2}{|c|}{ Medium } & Very_Large & Zero \\
\hline \multicolumn{4}{|c|}{ EX5 } & \multicolumn{4}{|c|}{ EX6 } & \multicolumn{3}{|c|}{ EX7 } \\
\hline \multicolumn{2}{|c|}{ Values of $\boldsymbol{I}$} & \multicolumn{2}{|c|}{ Values of $\mathbf{N}$} & & Values of $\mathbf{I}$ & \multicolumn{2}{|c|}{ Values of $\mathbf{N}$} & \multicolumn{2}{|c|}{ Values of I } & Values of $N$ \\
\hline Null & & \multicolumn{2}{|c|}{ Very_Large } & \multicolumn{2}{|c|}{ Null } & \multicolumn{2}{|c|}{ Zero } & \multicolumn{2}{|c|}{ Null } & Zero \\
\hline Zero & & Very_L & arge & Zer & & Zero & & Zero & & Medium \\
\hline Small & & Large & & Sma & all & Small & & Small & & uarge \\
\hline Medium & & Large & & Mec & dium & Medium & & Mediun & & arge \\
\hline Large & & Medium & & Lar & rge & Large & & Large & & Very_Large \\
\hline Very_Large & & Zero & & Ver & ry_Large & Very_Large & & Very_L & Large & Very_Large \\
\hline
\end{tabular}

